Hernández Breña, W. Gerencialismo en un ambiente de regulaciones débiles y cultura fuerte: El caso de las Fiscalías Corporativas en Perú. Derecho y Ciencias Sociales. Octubre 2017. № 17. (Estudios actuales sobre la justicia penal) Pgs145-163 ISNN 1852-2971. Instituto de Cultura Jurídica y Maestría en Sociología Jurídica. FCJ y S. UNLP

\title{
Gerencialismo en un ambiente de regulaciones débiles y cultura fuerte: El caso de las Fiscalías Corporativas en Perú
}

\author{
Managerialism under weak regulations and strong organizational culture: The case of \\ Prosecutors in charge of corruption cases in Peru
}

Wilson Hernández Breña

\section{Resumen}

En el Perú, la corporativización llegó en el 2011a las Fiscalías Especializadas en Delitos de Corrupción de Funcionarios (en adelante, FC Corporativas Corporativas), luego de un supuesto éxito en la jurisdicción civil. Priorizando la eficiencia por encima de la calidad, esta medida gerencialista planteó compartir tareas administrativas y jurídicas entre los distintos fiscales, bajo el liderazgo de un fiscal coordinador. En los hechos, la corporativización estuvo lejos de alcanzar la eficiencia. En este artículo, analizamos cómo la generalidad de las reglas establecidas para el funcionamiento de las FC Corporativas y los rasgos de una cultura fiscal que desvaloriza lo corporativo en forma insidiosa, crearon incentivos que generaron la aparición de cuatro estilos de gestión directamente influenciados por dos características delos fiscales coordinadores (comunicación y proactividad). Asumiendo que la cultura fiscal crea nuevos incentivos, especialmente en escenarios de transición (hacia la corporativización y hacia la adopción de un nuevo Código Procesal Penal), no exaltamos la cultura ni damos única cabida a una mejor regulación como eje de solución. Sostenemos que la comprensión de la interacción entre regulaciones y cultura fiscal brinda un mejor espacio de trabajo para políticas públicas que busquen la eficiencia pero también la calidad del trabajo de los fiscales.

Palabras Clave: Cultura; fiscalías; gestión; reglas

\begin{abstract}
In 2011, Peruvian General Prosecutors in charge of corruption cases were clustered, after successful previous experiences in the civil jurisdiction. Clustering works by making efficiency -not quality-a priority and sharing administrative and investigation tasks among prosecutors led by a coordinator. In practice, clustering rarely achieved efficiency. In this article, we analyze how vague clustering regulations and an organizational culture that disregarded clustering created incentives that fostered four management styles influenced by two coordinators' characteristics (communication and proactivity). Knowing that organizational culture creates incentives, especially in times of transition (like the clustering and the adoption of a new Code of Criminal Procedures), we don't overestimate culture nor better regulations as solutions. It is the interaction between them what offers a better space for public policies seeking efficiency but also quality in General Prosecutors' Office work.
\end{abstract}

Keyword: Culture; General prosecutors; management; regulations

\footnotetext{
•Universidad de Lima.

Recibido: 29/06/2017 Aceptado con correcciones: 09/09/2017
} 
Hernández Breña, W. Gerencialismo en un ambiente de regulaciones débiles y cultura fuerte: El caso de las Fiscalías Corporativas en Perú. Derecho y Ciencias Sociales. Octubre 2017. № 17. (Estudios actuales sobre la justicia penal) Pgs145-163 ISNN 1852-2971. Instituto de Cultura Jurídica y Maestría en Sociología Jurídica. FCJ y S. UNLP

\section{Gerencialismo en un ambiente de regulaciones débiles y cultura fuerte: El caso de las Fiscalías Corporativas en Perú}

Wilson Hernández Breña

\section{Introducción}

Desde mediados de los noventa, la reforma de la justicia en el Perú tomó un fuerte componente de gestión. En la justicia penal, este énfasis adquirió más fuerza en el 2006 con la implementación de un nuevo Código Procesal Penal que dejó atrás el sistema inquisitivo por uno oral. Detrás de estos cambios, se introdujo un modelo de justicia gerencial diseñado para lo público pero ligado en lo operativo a la lógica privada del management(González, 2016).

Desde entonces, algunas iniciativas han sido recurrentes, como la reducción de la carga administrativa de los jueces, la creación de administradores de juzgados, y la introducción de servicios y recursos compartidos entre juzgados o entre fiscales a fin de crear unidades corporativas. Este artículo se concentra en esta última iniciativa y en una jurisdicción particular: las FC Corporativas.

La corporativización, ya implementada con supuesto éxito en la jurisdicción civil en el Perú, se aplicó desde el 2011en las FC Corporativas. En este ámbito, la corporativización se diseñó como la capacidad de compartir las diligencias preliminares, planificar estrategias conjuntas, investigar un caso en equipo, generar la especialización en alguna de las etapas de la investigación y del proceso judicial, crear esquemas de reemplazo en diligencias cuando el fiscal asignado no pueda asistir, entre otras actuaciones factibles de ser realizadas en forma cooperativa y coordinada.

En el contexto latinoamericano, Duce (2008) señala que las reformas procesales penales tuvieron un impacto profundo en la reconfiguración institucional del Ministerio Público. Pese a ello, ha sido una institución poco estudiada en América Latina, sobre todo en el contexto de la reforma procesal penal.

Nuestro interés estuvo en analizar cómo la generalidad de las reglas para el funcionamiento de las FC Corporativas y los rasgos de una cultura fiscal que insidiosamentedesvaloriza lo corporativo, generaron incentivos para la aparición espontánea de estilos de gestión basados en reglas informales íntimamente ligadas a dos características de los fiscales coordinadores (comunicación y proactividad). De esta forma, identificamos cuatro estilos de gestión: 
Hernández Breña, W. Gerencialismo en un ambiente de regulaciones débiles y cultura fuerte: El caso de las Fiscalías Corporativas en Perú. Derecho y Ciencias Sociales. Octubre 2017. № 17. (Estudios actuales sobre la justicia penal) Pgs145-163 ISNN 1852-2971. Instituto de Cultura Jurídica y Maestría en Sociología Jurídica. FCJ y S. UNLP

proactivo, administración abierta, desorganización con dinámica y organización lotizada pura.En la práctica, aun cuando la corporativización apuntó a mejorar la eficiencia, en la mayoría de los estilos de gestión se obtuvo lo contrario.

Dos supuestos sostienen nuestra argumentación. En primer lugar, asumimos que la cultura fiscal juega un rol central en la creación de incentivos que, a su vez, alteran las prácticas cotidianas de gestión que realizan los fiscales. No consideramos que estas prácticas, asentadas en una cultura fiscal bastante definida, respondan a una lógica economicista de costos y beneficios. Más bien, creemos que responden a una lógica no económica de incentivoscon doble sentido: son incentivos frente a los cuales los fiscales reaccionan y dicha reacción está íntimamente vinculada al grado de comunicación y proactividad de los fiscales coordinadores. En segundo lugar, asumimos que bajo escenarios de transición las reglas informales tienen la capacidad de reemplazar, socavar o reformar las reglas formales al margen de cuál sea el peso de estas últimas (Grzymala-Busse, 2010).Al respecto, las FC Corporativas estuvieron inmersas no en uno sino en dos procesos de transición simultáneos: su corporativización y la adopción de un nuevo Código Procesal Penal.

En nuestra argumentación, el nexo entre gestión y cultura fiscal es central, aunque no evidente. Los estudios cuantitativos que estudiaron el por qué del fracaso de las reformas judiciales apuntaron a la cultura como el gran factor explicativo (Garavano, 2004). Resultados de este tipo impulsaron a que la introducción del nuevo Código Procesal Penal en Panamá sea acompañada por medidas destinadas a crear una nueva cultura y prácticas de gestión a fin de evitar malas prácticas, corrupción y resistencia al cambio (Centro de Estudios de la Justicia de las Américas, 2014). Sin embargo, para Ciocchini (2013), la atribución de responsabilidad a la cultura fiscal nace como conclusión de la existencia de factores que no pueden ser racionalizados por la lógica de costo y beneficio del análisis económico empleado en dichos estudios.

Hasta 1980, en el Perú las funciones que ahora son del Ministerio Público (también llamada Fiscalía de la Nación) eran competencia del Poder Judicial. En ese año, se crea el Ministerio Público como un organismo autónomo. Las FC Corporativas son fiscalías especializadas. Solo reciben denuncias de corrupción de funcionarios, en su mayoría relacionadas a los delitos de peculado y colusión. Cada fiscal tiene una carga que varía entre 25 y 57 casos. Cada FC Corporativa está compuesta por varias fiscalías, pero es liderada por un único fiscal, denominado fiscal coordinador. En cada fiscalía, existen dos niveles de fiscal: el provincial y, bajo su jerarquía, fiscales adjuntos provinciales. A fin de evitar complicaciones, a los primeros se les denominará simplemente fiscales y a los fiscales adjuntos provinciales se les 
Hernández Breña, W. Gerencialismo en un ambiente de regulaciones débiles y cultura fuerte: El caso de las Fiscalías Corporativas en Perú. Derecho y Ciencias Sociales. Octubre 2017. № 17. (Estudios actuales sobre la justicia penal) Pgs145-163 ISNN 1852-2971. Instituto de Cultura Jurídica y Maestría en Sociología Jurídica. FCJ y S. UNLP

llamará fiscales de segundo nivel. Un fiscal tiene entre dos a cuatro fiscales de segundo nivel a su cargo.Las denuncias se derivan al fiscal y este luego las deriva al fiscal de segundo nivel que considere pertinente. Para esta investigación, fueron entrevistados 41 de 97 fiscales de cuatro distritos fiscales (circunscripción similar a una región) más representativos (Lima, Áncash, Ayacucho y Junín). Las entrevistas se realizaron entre diciembre del 2013 y enero del siguiente año.Cada entrevista duró entre treinta y noventa minutos, y se realizaron siempre en las oficinas de cada fiscal. Por lo general, las oficinas eran cerradas y permitieron la debida confidencialidad del caso.

\section{Una regulación muy general}

A diferencia del antiguo modelo penal inquisitorio, en el nuevo Código Procesal Penal cada FC Corporativacuenta con un coordinador. A fin de ordenar su trabajo como gestor, sus funciones fueron reguladas bajo la Resolución Administrativa 242-2007-MP-FN. En este documento, se precisó que las regulaciones dadas tenían por finalidad "afinar el trabajo administrativo y operativo de los despachos fiscales dentro del nuevo modelo procesal penal". El Reglamento es la fuente para el trabajo del fiscal coordinador y de las FC Corporativas, pero también es fuente de problemas. Son dos sus debilidades centrales. Primero: su generalidad. Posee reglas generales que incentivan su aplicación indebida y crean una amplia discrecionalidad sobre cómo aplicarlas, lo que al mismo tiempo generaque la gestión entre coordinador y coordinador sea muy diferente. Segundo, el Reglamento se limita a aspectos bastante operativos con bajo grado de aporte a la corporativización.

Aun cuando lo que se buscó con un fiscal coordinador fue mejorar la eficiencia en el funcionamiento de las FC Corporativas, en la práctica las debilidades del Reglamento incidieron en que la figura del coordinador se restrinja a la de un administrador tradicional centrado en funciones poco productivas bajo una lógica de corporativización. Ello, por ejemplo, minimizó la posibilidad de reglamentar la coordinación de diligencias, estrategias de investigación y criterios de archivamiento de denuncias.

Para efectos analíticos, podemos clasificar las funciones de los fiscales coordinadores en tres grupos: control y reporte, administración de personal y administración operativa.

En la mayoría de casos, el Reglamento define las funciones de control y reporte de los coordinadores con escasa precisión. Esto no lo convierte necesariamente en un Reglamento deficiente, pero sí deja un espacio de discrecionalidad para el que fiscales, preparados para investigar y no para gestionar, no están preparados. Por ejemplo, el Reglamento señala que la 
Hernández Breña, W. Gerencialismo en un ambiente de regulaciones débiles y cultura fuerte: El caso de las Fiscalías Corporativas en Perú. Derecho y Ciencias Sociales. Octubre 2017. № 17. (Estudios actuales sobre la justicia penal) Pgs145-163 ISNN 1852-2971. Instituto de Cultura Jurídica y Maestría en Sociología Jurídica. FCJ y S. UNLP

función de los coordinadores es gestionar los despachos fiscales penales corporativos, para una eficaz y eficiente aplicación del nuevo modelo procesal penal. Lo negativo de esta fórmula es su generalidad, y lo negativo es que ningún fiscal, limitado por sus propias capacidades de gestión, la aprovechó para implementar cambios importantes.

Las funciones de control y reporte son generales, aparentemente fijadas así para brindar a los coordinadores un marco amplio que no los limite a un escaso conjunto de información por reportar. Son tres las funciones de control y reporte: informar periódicamente sobre todas las acciones y resultados del funcionamiento de las fiscalías corporativas alos fiscales superiores; informar al fiscal superior acerca del incumplimiento de directivas e instrucciones de parte de los fiscales; y proveer informes periódicos al equipo técnico del Ministerio Público sobre el avance de la implementación del nuevo Código Procesal Penal. Mientras que la primera función tiene un matiz de control y sanción, las otras dos buscan medir eficiencia. El problema es que, como sucede con otras instituciones del sistema de justicia, rara vez se mide la eficiencia del desempeño fiscal en forma adecuada. La cultura de reporte estadístico tiene arraigo. Se emiten anualmente informes de ese tipo, pero estos carecen de criterio sobre cómo construir indicadores útiles para la gestión fiscal. Tareas de este tipo terminan en reportes estadísticos generales sobre el estado de los casos (cantidad de casos, número de denuncias archivadas, etc.), dejando de lado la posibilidad de conocer la cantidad de diligencias programadas y realizadas, el porcentaje de manifestaciones frustradas (porque no acudió la persona citada), etc.

La administración del personal es la segunda función de los coordinadores. Deben controlar la asistencia y permanencia del personal, tarea que comparten con el órgano de control interno del Ministerio Público. Como señaló un fiscal coordinador, son tareas tediosas que quitan tiempo valioso que bien podría ser redirigido a tareas más productivas.

El tercer tipo de función es la administrativo-operativa. Las FC Corporativas deben realizar la asignación de denuncias que llegan a cada fiscal así como hacerle seguimiento, especialmente a los casos más importantes o emblemáticos. Esto último, según el Reglamento, se hace de acuerdo al criterio del fiscal coordinador o de su superior. Nuevamente, el Reglamento opta en este punto por ser general, pero dejando una discrecionalidad amplia que en la práctica ha sido cubierta en forma poco clara y predecible. Si bien existen los lineamientos de política del Ministerio Público que instan a que la distribución de las denuncias entre fiscales sea equitativa a fin de evitar fiscalías sobrecargadas de casos simples o complejos, el Reglamento no desarrolló los criterios para efectuar tal distribución. Los criterios quedaron en manos de cada fiscal coordinador. Pero, al carecer de guías técnicas para tal función, los criterios 
Hernández Breña, W. Gerencialismo en un ambiente de regulaciones débiles y cultura fuerte: El caso de las Fiscalías Corporativas en Perú. Derecho y Ciencias Sociales. Octubre 2017. № 17. (Estudios actuales sobre la justicia penal) Pgs145-163 ISNN 1852-2971. Instituto de Cultura Jurídica y Maestría en Sociología Jurídica. FCJ y S. UNLP

resultaron ser poco técnicos, distintos entre fiscales coordinadores e incluso, como señaló un fiscal, poco transparentes. Otro fiscal señaló que las denuncias complejas se distribuyen al fiscal que muestra algún interés en esta, al margen de si antes vio o no casos similares.

En suma, la figura del fiscal coordinador se acerca más a la de un administrador que a la de un coordinador de órgano corporativo. Sus funciones generales deben ser adaptadas a las necesidades de coordinación, pero no están libres de otras influencias como las que provienen de la cultura fiscal.

\section{La cultura fiscal}

En el Perú, la cultura judicial ha sido un objeto de estudio poco común. Menos aún lo ha sido la cultura fiscal. León (1996) señala que el Poder Judicial peruano es una institución formalista, de relativa independencia y jerárquica. Podríamos extender esta caracterización al Ministerio Público sin temor a brindar un diagnóstico equivocado. Para efectos de este trabajo, nos importa partir de la jerarquía. El Ministerio Público es una entidad jerárquica por norma y por cultura. Su Ley Orgánica establece que los fiscales forman parte de un cuerpo jerárquicamente organizado y como parte de éste deben sujetarse a las instrucciones impartidas por sus superiores. La vía regular instaurada para la comunicación entre físcales de distinto nivel ha sido la queja (apelación de alguna decisión fiscal) y no una vía de gestión (reuniones de coordinación).

La jerarquía no solo es un factor constituyente del Ministerio Público y sus fiscales, sino también un factor que, asimilado y reproducido por sus fiscales, constituye a la institución. Nos interesa plantear la relación entre esa cultura y su relación con la aparición espontánea de estilos de gestión. El nexo está en tres rasgos: (i) la exacerbación del principio de autonomía y el bloqueo que esto ejerce a la introducción de cambios de gestión que alteran la forma en que cada fiscal administra sus recursos, procesos y personal; (ii) la aplicación del principio de jerarquía en el Ministerio Público como uno de sujeción y menos como uno de coordinación; y (iii) la visión de política pública individualizada (y no transversal) que existe en los fiscales y que se da como consecuencia de la lógica de trabajo "caso por caso" en que se basa el razonamiento y el trabajo fiscal.

La autonomía, primer rasgo que analizaremos, es un principio central en la administración de justicia. Pero al mismo tiempo es un principio que genera muchas sensibilidades, especialmente en contextos donde hubo intromisión política en el sistema de justicia. Ese fue 
Hernández Breña, W. Gerencialismo en un ambiente de regulaciones débiles y cultura fuerte: El caso de las Fiscalías Corporativas en Perú. Derecho y Ciencias Sociales. Octubre 2017. № 17. (Estudios actuales sobre la justicia penal) Pgs145-163 ISNN 1852-2971. Instituto de Cultura Jurídica y Maestría en Sociología Jurídica. FCJ y S. UNLP

el caso de la reforma judicial peruana de los noventa y su contribución a lo que acá llamamos la exacerbación del principio de autonomía.

Dentro de límites adecuados, el principio de autonomía marca el espacio de independencia y criterio fiscal para una decisión independiente. Sin embargo, fuera de este espacio de decisión, la autonomía tiene menos sentido. Nos referimos a la extensión de la autonomía al ámbito de la gestión fiscal.

En forma frecuente, la gestión fiscal ha sido vista como un espacio más de la autonomía del fiscal. Alterarlo intencionadamente ha sido tomado como una posibilidad de injerencia. Como señala Duce (2008, pág. 75) para el caso latinoamericano, los fiscales gozan de un importante nivel de autonomía para organizar su trabajo y tomar decisiones acerca de la persecución penal, “[...] sin poder aceptar instrucciones o directivas acerca de cómo llevar adelante su trabajo”. Mientras que la falta de atención a necesidades generales (presupuesto, número de fiscalías, personal, etc.) es tomada como una vulneración de la autonomía, la solución de carencias de gestión más puntuales orientadas al logro de la eficiencia (reorganización de las tareas administrativas que realiza el fiscal) también es vista como una vulneración a este principio.

El segundo rasgo de la cultura fiscal de interés para nuestra argumentación es el que se forja alrededor del principio de jerarquía. Este principio, establecido en la Ley Orgánica del Ministerio Público, tiene dos características particulares: brinda estructura y poder socializador.

De un lado, brinda estructura porque, en su artículo $5^{\circ}$ la Ley Orgánica establece que los fiscales forman parte de un cuerpo jerárquicamente organizado y que, como tal, deben sujetarse a las instrucciones impartidas por sus superiores. En una institución donde la jerarquía y la sujeción son reglas escritas de una cultura viva, han sido los canales de comunicación internos para la formación y discusión de decisiones los que han visto limitadas sus posibilidades de desarrollo. La comunicación se ha dado principalmente a través del canal unidireccional formal de manifestación de ideas entre fiscales: las apelaciones que someten la posición de un fiscal ante un superior. Esta lógica se valida con la existencia de otros principios procesales (como el de la doble instancia) que buscan garantizar valores mayores como la imparcialidad de la justicia.

Del otro lado, el principio de jerarquía tiene un poder socializador. Los fiscales lo reconocen y lo reclaman como un principio central en sus funciones. Y así lo es. Sin embargo, mientras esa socialización privilegia canales con sesgo unidireccional con un espacio de discusión bastante limitado, obvia otras formas de comunicación presentes en cualquier otra 
Hernández Breña, W. Gerencialismo en un ambiente de regulaciones débiles y cultura fuerte: El caso de las Fiscalías Corporativas en Perú. Derecho y Ciencias Sociales. Octubre 2017. № 17. (Estudios actuales sobre la justicia penal) Pgs145-163 ISNN 1852-2971. Instituto de Cultura Jurídica y Maestría en Sociología Jurídica. FCJ y S. UNLP

organización. Las apelaciones son comunicaciones limitadas, enmarcadas en el formalismo de los dictámenes fiscales y regladas por la imposición del juicio del superior. No representan una comunicación en el sentido de hacer común una idea entre grupos. En otras palabras, el principio de jerarquía se vive más como sujeción y menos como coordinación.

Justamente, buscando ampliar el horizonte impuesto por una lectura rígida del principio de jerarquía, el Tribunal Constitucional ensayó relacionar dicho principio a su efecto sobre la política pública. Reconociendo que la política pública es el resultado de un cúmulo de decisiones individuales, para el máximo intérprete de la Constitución dicha política debe nutrirse de la coordinación entre fiscales:

“[...] de acuerdo con el artículo 5. ${ }^{\circ}$ de la Ley Orgánica del Ministerio Público, se reconoce también un principio de jerarquía, según el cual los Fiscales pertenecen a un cuerpo jerárquicamente organizado y deben sujetarse a las instrucciones que les impartan sus superiores. Tal disposición, si se quiere que sea conforme a la Constitución, sólo se justifica si de lo que se trata es de dotar de coherencia y unidad al ejercicio de las funciones constitucionales que establece el artículo $159 .^{\circ} \mathrm{de}$ la Constitución. De ahí la necesidad de que se establezcan también relaciones de coordinación conjunta entre los Fiscales de los distintos niveles, en atención a que la política de persecución criminal no puede ser definida por cada fiscal o juez en particular, pues ello corresponde al propio Estado."

El tercer rasgo de la cultura fiscal es el que favorece una visión de política pública individualizada, no transversal. Las instituciones como el Poder Judicial y el Ministerio Público funcionan privilegiando la visión del caso (árbol) y no de la problemática global detrás de ellos (bosque). El fiscal trabaja caso por caso. En términos de Binder (s/f), los fiscales siguen viendo la reforma procesal penal y sus cambios como un problema procesal y jurídico en lugar de hacerlo como uno de políticas públicas y, en consecuencia, de contenido político y consecuencias sociales. Si bien los fiscales generan precedentes a partir de sus productos (dictamen, archivo, etc.), tienen dificultades para sistematizar el insumo que reciben (denuncias) e identificar patrones de denuncias (formas de corrupción específicas) pasibles de ser investigadas en forma similar (protocolizar). Salvo excepcionalmente, la lógica del caso por caso produce investigaciones y decisiones con menor probabilidad de ser iguales a casos previos investigados por el mismo fiscal o similares a lo investigado por otros fiscales. 
Hernández Breña, W. Gerencialismo en un ambiente de regulaciones débiles y cultura fuerte: El caso de las Fiscalías Corporativas en Perú. Derecho y Ciencias Sociales. Octubre 2017. № 17. (Estudios actuales sobre la justicia penal) Pgs145-163 ISNN 1852-2971. Instituto de Cultura Jurídica y Maestría en Sociología Jurídica. FCJ y S. UNLP

\section{Estilos espontáneos de gestión}

En este marco reglas formales débiles y de una organización afectada por su cultura, la capacidad de organizarse se vuelve menos institucional y adquiere un tono más individual. El riesgo es que al apartarse de las reglas establecidas, se abren incentivos no previstos. Pero esos incentivos tienen el riesgo de ser incompatibles con el objetivo detrás de las funciones planificadas para las FC Corporativas. Tales incentivos siempre estarán conectados a las posibilidades de cada persona, en la medida que tales posibilidades se configuran a partir de preferencias individuales, pero resultan siendo poco conocidas por quienes crean las reglas (Dasgupta, Hammond, \& Maskin, 1979). En otras palabras, el resultado de la generalidad de las reglas y la cultura fiscal generó resultados no esperados. Los fiscales buscaron sus propias formas de gestión a fin de cumplir el mandato de las funciones corporativas. En ese objetivo, identificamos lo que hemos llamado estilos espontáneos de gestión.

Son espontáneos en el sentido de que derivan el impulso propio (no institucional) del fiscal coordinador, pero eso no quiere decir que carezcan de estructura. Por el contrario, estos estilos son altamente dependientes de dos características del fiscal coordinador: el nivel de comunicación que ha tejido en la FC (baja o alta) y su proactividad (baja o alta). La combinación de ambas categorías da pie a la aparición de cuatro estilos de gestión: proactivo, administración abierta, organización lotizada pura y desorganización con dinámica. Aunque las FC Corporativas fueron creadas para ganar eficiencia, la mayoría de estos estilos condujeron a resultados opuestos.

En la descripción de los estilos espontáneos de gestión que sigue a continuación, se dará énfasis a dos temas: la discusión de aspectos jurídicos (pautas para las investigaciones, difusión y discusión de normativa y jurisprudencia, etc.) y administrativos (distribución de recursos), y la corporativización.

Antes de describir cada estilo de gestión, es necesario hacer tres precisiones. Primero, una FC Corporativa no tiene un estilo puro, pero sí por lo general un estilo predominante. Cada estilo no es una categoría estanca, sino una zona de grises con transiciones poco claras. Segundo, no se trata de estilos teóricos, sino fácticos. De ahí que no se trate necesariamente de estilos óptimos. Tercero, la transición de un estilo a otro depende del fiscal coordinador, pero también de aspectos estructurales en los que el fiscal no tiene ninguna influencia (cantidad y calidad del personal, apoyo logístico, presupuesto, etc.) así como externos a éstos (políticas anticorrupción, jurisprudencia, desarrollo normativo, etc.). 
Hernández Breña, W. Gerencialismo en un ambiente de regulaciones débiles y cultura fuerte: El caso de las Fiscalías Corporativas en Perú. Derecho y Ciencias Sociales. Octubre 2017. № 17. (Estudios actuales sobre la justicia penal) Pgs145-163 ISNN 1852-2971. Instituto de Cultura Jurídica y Maestría en Sociología Jurídica. FCJ y S. UNLP

\section{Figural: Estilos espontáneos de gestión}

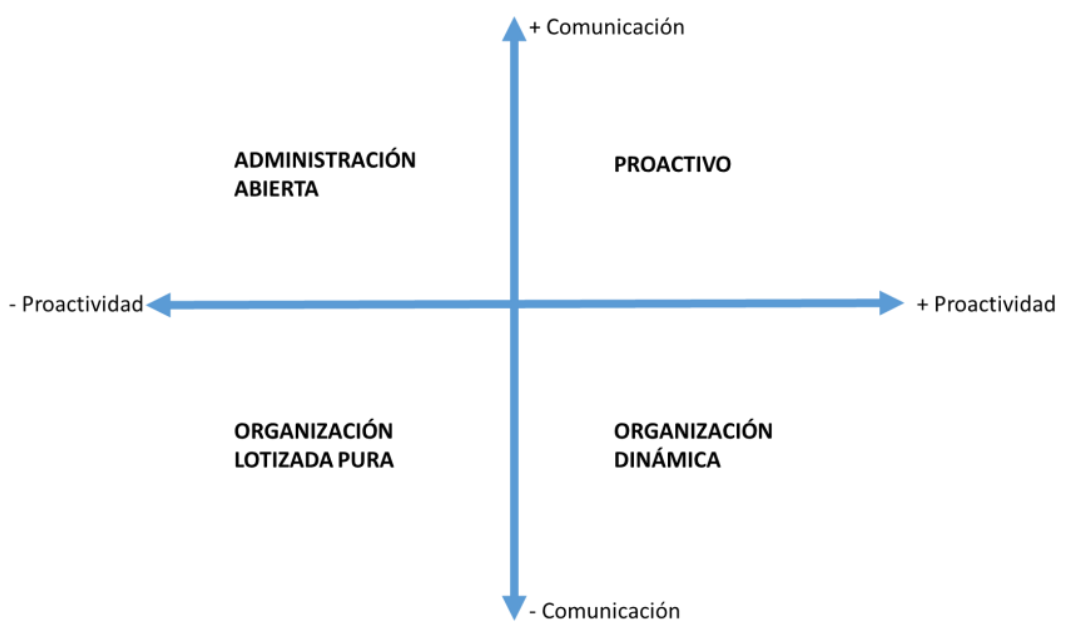

Elaboración propia.

\subsection{Estilo proactivo}

Es el estilo de coordinación con mejor performance, sin que ello signifique un rendimiento óptimo. Una de las características centrales de este estilo es la institucionalización de acuerdos y de espacios de ensayo.

La institucionalización de acuerdos está basada en reuniones. Son reuniones de convocatoria abierta. Participan no solo los fiscales coordinadores, sino también fiscales de segundo nivel. Esto tiene sentido porque en tales reuniones se discuten acuerdos relacionados a las investigaciones en marcha que todos esos fiscales están trabajando. Se discuten las pautas para realizar diligencias, estrategias, resoluciones de jueces del mismo distrito fiscal, sentencias de la Corte Suprema, etc. Las reuniones producen acuerdos escritos, en ocasiones institucionalizados como directivas y en otros casos como documentos de menor rango. En ocasiones, incluso los acuerdos son verbales. De acuerdo a los fiscales, esta práctica ha legitimado las reuniones y ha generado compromiso para discutir y aplicar los acuerdos tomados.

La institucionalización de espacios de ensayo se refierea la realización de simulaciones de juicio oral.Son pocos los casos que llegan a dicha instancia (4\%) pues, según datos del Ministerio Público, la tasa de archivo de denuncias es alta (entre 60\% y $80 \%$ ) al igual que las salidas intermedias que evitan que el caso pase a juicio oral. La escasa oportunidad de que un fiscal llegue a juicio oral, reduce su performance y posibilidad de éxito, sobre todo teniendo en cuenta que se trata de audiencias orales bajo un sistema relativamente nuevo. A fin de suplir esta falta de experiencia, las simulaciones de juicio oral se realizan una o dos semanas 
Hernández Breña, W. Gerencialismo en un ambiente de regulaciones débiles y cultura fuerte: El caso de las Fiscalías Corporativas en Perú. Derecho y Ciencias Sociales. Octubre 2017. № 17. (Estudios actuales sobre la justicia penal) Pgs145-163 ISNN 1852-2971. Instituto de Cultura Jurídica y Maestría en Sociología Jurídica. FCJ y S. UNLP

antes del juicio oral real. La simulación enfrenta al fiscal que irá al juicio oral con el resto de fiscales que fungen de abogados contrincantes. Durante las simulaciones, las estrategias de estos últimos varían entre la argumentación que busca enfrentar argumento contra argumento y la performance del otro abogado en el juicio oral (preguntas sin sentido, interrupciones, ridiculizaciones, etc.). Ante la falta de mejores esquemas de formación, las simulaciones son bien valoradas por los fiscales.

"Entre nosotros mismos nos hemos capacitado para las audiencias. Me pongo como el peor abogado, trato de ridiculizarlo, de tal forma. Me acuerdo de la doctora[la fiscal], no la dejé ni hablar. Al día siguiente, al abogado le aplicó lo mismo. Sugestiva, capciosa, sugerente. Y un detalle: El fiscal al que le va a ir bien es el que se reponga cuando pierda, no el que gana todos los casos.” (Fiscal de Ayacucho).

La institucionalización de las prácticas descritas adelanta la existencia de mejores condiciones para la corporativización. Aunque esta ha sido bastante puntual, sí se la ha observado en la realización de ciertas diligencias.Bajo el estilo proactivo, la corporativización respondió a un dilema cotidiano (cómo reasignar recursos) mediante la transformación (temporal y funcional) de una organización jerárquicaen una matricial. Bajo esta transformación, cada fiscal mantiene la responsabilidad por sus casos, pero al mismo tiempo puede formar parte de los equipos de apoyo de otros fiscales a fin de realizar ciertas diligencias (incautaciones y constituciones), puede ser reemplazado por sus pares ante la imposibilidad de acudir a una diligencia, o puedehacer uso de determinados recursos escasos (peritos y movilidad) en forma coordinada con el resto de fiscales (evitando que un fiscal cargue de demasiado trabajo al perito o que limite la disponibilidad de la movilidad cuando otros fiscales la necesiten).

Por más interesantes que sean todas estas prácticas, están lejos de reflejar una corporativización profunda. Los mismos fiscales señalan que muchas diligencias se hacen en forma individual (como solicitar documentación a determinadas instituciones), aun cuando sería mucho más lógico ejecutarlas en forma conjunta (enviar una solicitud masiva de información). Es probable que lo que acá hemos denominado estilo de gestión proactiva sea un estilo que cueste tiempo establecer pues, basado en la comunicación y proactividad, necesita fiscales líderes. Y de esos se observaron muy pocos.

\subsection{Estilo de administración abierta}


Hernández Breña, W. Gerencialismo en un ambiente de regulaciones débiles y cultura fuerte: El caso de las Fiscalías Corporativas en Perú. Derecho y Ciencias Sociales. Octubre 2017. № 17. (Estudios actuales sobre la justicia penal) Pgs145-163 ISNN 1852-2971. Instituto de Cultura Jurídica y Maestría en Sociología Jurídica. FCJ y S. UNLP

Este estilo es el resultado de la buena comunicación del coordinador junto con su baja proactividad. La función de corporativización en este estilo es la consecuencia plena del repliegue del fiscal coordinador como articulador proactivo de intereses. Es debido a esto que la institucionalización se da con mayor intensidad en aquellas prácticas donde la legitimidad del coordinador es menos necesaria, es decir, en los aspectos administrativos.

La administración abierta deja de lado la institucionalización de acuerdos. Sí los hay, pero generalmente son verbales y, por tanto, con limitado peso para ser monitoreados en el tiempo. Salvo excepciones como la discusión de jurisprudencia o nuevas normas, los físcales no llevan las problemáticas jurídicas de sus casos a las reuniones de coordinación, ya que estas privilegian temas administrativos. Bajo este estilo, se debilitan los espacios de discusión pues si bien los fiscales que no asistieron pueden revisar las pocas actas que se producen, al mismo tiempo los fiscales de menor jerarquía están excluidos de estos espacios, aunque según un fiscal tal exclusión se justificaría en el capital de este estilo: la necesidad de conservar una mejor comunicación entre pocas personas.

"Compartir formas de investigar entre fiscales, eso sería útil. Las reuniones semanales se han implementado hace dos meses, y en estos tres meses quien va es el fiscal provincial, no los adjuntos[fiscales de segundo nivel]. Debería haber conversaciones aquí, pero no entre muchos o al menos no para empezar porque cuántos más integrantes la comunicación es menor. Se debe empezar con el trabajo corporativo en el propio despacho y luego buscar reuniones entre fiscalías corporativas" (Fiscal de Lima).

La pérdida del espacio de discusión institucional no ha hecho desaparecer la necesidad de discusión. Más bien, esta necesidad se ha trasladado a otros campos: uno más cerrado (al interior de cada fiscalía, donde el fiscal coordina con sus fiscales de segundo nivel) y otro de tipo informal que se alimenta de la cercanía o amistad entre fiscales (conversaciones "de pasillo"). Mientras que en el campo cerrado, las discusiones pueden ganar profundidad y peso para generar acuerdos entre algunos pocos fiscales, el campo informal se advierte como inestable en tanto no solo carece de un espacio institucionalizado sino también de estructura temporal. 
Hernández Breña, W. Gerencialismo en un ambiente de regulaciones débiles y cultura fuerte: El caso de las Fiscalías Corporativas en Perú. Derecho y Ciencias Sociales. Octubre 2017. № 17. (Estudios actuales sobre la justicia penal) Pgs145-163 ISNN 1852-2971. Instituto de Cultura Jurídica y Maestría en Sociología Jurídica. FCJ y S. UNLP

"En el despacho fiscal tenemos 'plenitos', esto es, reuniones entre los fiscales de un mismo despacho para unificar criterios o discutir cuestiones de casuística que nos están complicando la vida." (Fiscal de Lima).

"Hay más comunicación por un tema generacional que por un tema funcional, no es por el diseño en sí. Es porque hay ciertas simpatías. Pero sí hay comunicación fluida.” (Fiscal de Lima).

"Hace poco un fiscal de este despacho participó en declaraciones tomadas por otro despacho. Este depende mucho, claro, de que los fiscales provinciales se lleven bien entre ellos, que coordinen para que se presten apoyo. Esto es impensado en las [fiscalías] comunes [no corporativas]. No solo funciona con audiencias sino diligencias de todo tipo." (Fiscal de Lima).

Bajo el estilo de administración abierta, el esfuerzo de corporativización se gesta por la necesidad o presión. Puede haber impulso del fiscal coordinador, pero es más reactivo que proactivo. Se da en la necesidad de investigar casos complejos o emblemáticos que requieren realizar diligencias de mayor despliegue del que un fiscal solitario puede realizar o se dan por la presión mediática en un caso mediatizado. En estos casos, la corporativización representa una respuesta a la urgencia de actuar en forma conjunta (pues no habría otra forma de hacerlo), en lugar de ser la respuestade un sistema en el que, bajo ciertos supuestos, se activa la realización de diligencias compartidas.

En suma, este tipo de estilo implica corporativizarse sin coordinación. Su sostenibilidad está limitada a las redes informales que se creen, lo que resulta en un estilo bastante frágil-aunque funcional- para los fiscales que lo que, en realidad, requieren es aclarar dudas, recibir soporte en casos extremos o administrar su lote (fiscalía).

\section{Estilo de organización lotizada pura}

En este estilo, tanto la comunicación como la proactividad son bajas. La lotización de la organización está marcada por la desconexión entre fiscales, lo que causa la desarticulación de prioridades, preocupaciones e intereses.Como señaló un físcal, "al final del día, cada despacho termina siendo como una fiscalía [Ministerio Público] independiente". 
Hernández Breña, W. Gerencialismo en un ambiente de regulaciones débiles y cultura fuerte: El caso de las Fiscalías Corporativas en Perú. Derecho y Ciencias Sociales. Octubre 2017. № 17. (Estudios actuales sobre la justicia penal) Pgs145-163 ISNN 1852-2971. Instituto de Cultura Jurídica y Maestría en Sociología Jurídica. FCJ y S. UNLP

La desconexión entre fiscales calza con la falta de un fiscal coordinador líder, reclamo de algunos fiscales que no termina de ser paradójico. En esencia, tal reclamo sugiere que la organización lotizada pura no es un estilo deseable ni natural pese a ser el que caracteriza al resto de fiscalías del Ministerio Público y pese a ser el estilo en el que todos los fiscales han laborado previamente.

\footnotetext{
"Nos falta líderes. El coordinador no hace la labor de coordinador. Debería saber todo lo que pasa con todos los adjuntos. Menos aún en lo que le pasa con los adjuntos de esos despachos. No hay conexión directa. Si bien uno no tiene capacidad de ver todos los casos, al menos un jefe debería saber qué está haciendo con cada caso. El coordinador debería llegar más a quienes están llevando cada caso." (Fiscal de Lima).
}

La institucionalización de acuerdos es baja. Las reuniones de coordinación no integran a los fiscales de segundo nivel. Los acuerdos tomados son transmitidos ocasionalmente y en todo caso no dejan registro escrito, sino que se difunden gracias a la comunicación entre fiscales y sus fiscales de segundo nivel.

Al igual que el estilo anterior, el abordaje de los temas jurídicos desde la coordinación se hace para el nivel mínimo exigible: casos complejos y emblemáticos, o discusión de normatividad relevante. El contrapeso ante la falta de impulso a los temas jurídicos desde la coordinación se da en cada fiscalía. Ahí se conversan y discuten pautas de investigación y jurisprudencia. Pero esto no es homogéneo entre los fiscales. Algunos fiscales dejan a sus fiscales de segundo nivelcon una libertad total de plantear estrategias de investigación en un sistema que justamente busca lo opuesto.

"No sé si mis disposiciones están bien o mal. Si estoy equivocada no hay mecanismo de retroalimentación." (Fiscal de Lima).

Siendo un estilo lotizado, lo corporativo se ha intentado poco. Y aun cuando se han realizado diligencias compartidas, la corporativización no es considerada como tal. Es bastante sintomático que los esfuerzos corporativos se reduzcan ala condición de "ayuda". 
Hernández Breña, W. Gerencialismo en un ambiente de regulaciones débiles y cultura fuerte: El caso de las Fiscalías Corporativas en Perú. Derecho y Ciencias Sociales. Octubre 2017. № 17. (Estudios actuales sobre la justicia penal) Pgs145-163 ISNN 1852-2971. Instituto de Cultura Jurídica y Maestría en Sociología Jurídica. FCJ y S. UNLP

"Para mí el tema de reemplazos en audiencias no ha funcionado. Mis adjuntos sí me ayudan pero a veces me avisan a última hora. Pero con ellos si nos reemplazamos." (Fiscal de Lima).

La comunicación que tienen los fiscales con el coordinador no es del todo fluida y mucho menos aun la que existe entre los fiscales y sus fiscales de segundo nivel. Los canales de consulta son similares a los del estilo anterior (cercanía y amistades) y el uso de los recursos escasos (movilidad y peritos) no se coordina, provocando conflictos propios de actores que priorizan sus intereses individuales por encima de los del colectivo.

Superficialmente, el culpable del fracaso de la corporativización es la carga de trabajo, eterna y no siempre correcta justificación de la lentitud del sistema de justicia (Hammergren, 2003). Sin embargo, creemos que la sobrecarga de casos esconde problemas mayores: la dificultad para quebrar una cultura asentada en el trabajo individual del fiscal, su débil comprensión sobre cómo formar una política pública cohesionada a partir de productos elaborados individualmente por las partes de un colectivo y la débil definición de reglas para gestionar lo corporativo manteniendo un objetivo de calidad en el trabajo fiscal.

"Pero con otros despachos fiscales yo no veo realmente mucha coordinación, salvo reuniones semanales. Cada uno sigue metido en su caso, no hay trabajo en equipo salvo un mega caso y el Fiscal de la Nación diga los dos despachos trabajen juntos" (Fiscal de Lima).

"Una de las desventajas es que el modelo corporativo no se aplica tanto en la práctica. El trabajo es tan absorbente que lo corporativo es nominal, no se hace trabajo en equipo conjunto. Pueden hacerse conversaciones informales entre los fiscales, pero no se logra potenciar el trabajo en equipo para permitir esto de manera más trascendente." (Fiscal de Lima).

\section{Estilo de desorganización con dinámica}

Por definición, es un estilo en el que un fiscal coordinador proactivo se desenvuelve con bajas habilidades de comunicación. Esta combinación no se halló más que puntualmente en ciertas interacciones. Por ello, en nuestra exploración, ningún distrito fiscal tuvo este estilo como primario. Más bien, se manifestó como un estilo secundario. 
Hernández Breña, W. Gerencialismo en un ambiente de regulaciones débiles y cultura fuerte: El caso de las Fiscalías Corporativas en Perú. Derecho y Ciencias Sociales. Octubre 2017. № 17. (Estudios actuales sobre la justicia penal) Pgs145-163 ISNN 1852-2971. Instituto de Cultura Jurídica y Maestría en Sociología Jurídica. FCJ y S. UNLP

El manejo de la institución de acuerdos es similar a lo ya observado en la organización lotizada pura. La diferencia es la mayor proactividad, lo que genera mayores niveles de convocatoria en las reuniones. Los acuerdos son básicamente verbales. Por ejemplo, en estas reuniones rara vez se discuten estrategias de investigación, pese a ser una preocupación constante del fiscal coordinador. La discusión jurídica es instrumental. Se limita a la difusión de sentencias o normas de interés.

La baja comunicación mina los esfuerzos derivados de la proactividad. No hay líder sin buena comunicación, ni propuestas que fácilmente sean aceptadas y aprobadas por el resto de fiscales. La falta de liderazgo compromete la base para la construcción de la corporativización, tanto en lo que respecta a diligencias compartidas o la promoción de iniciativas adicionales que pueden correr el riesgo de ser transformadas o rechazadas.

"Lo que debo señalar es que el fiscal superior nos ha proporcionado un grupo de 5 jóvenes asistentes que supuestamente son un grupo de elite que nos va a ayudar en los casos emblemáticos para conseguir prisiones preventivas. Pero por ejemplo yo no estoy de acuerdo, porque este grupo debería ayudar en toda la Investigación Preparatoria. Estos jóvenes sí pueden ayudar y los casos grandes ameritan este trabajo. Ha sido muy difícil poder convencerlo para que amplie a ese tipo de funciones las labores del grupo de apoyo.” (Fiscal de Áncash).

Todas estas características se han visto en mayor o menor medida en los estilos anteriores, lo cual reafirma que el estilo de desorganización con dinámica es más un espacio intermedio o de transición entre otros estilos que un estilopuro.

\section{Conclusiones}

En este trabajo analizamos críticamente la corporativización de las fiscalías anticorrupción en el Perú. En el marco de una lógica gerencialista, la corporativización de estas unidades se vio como un camino a la eficiencia, descuidando los estándares de calidad. El resultado fue un sistema que solo en pocos casos logró eficiencia.

Dos aspectos son centrales para entender nuestra argumentación: la generalidad de las reglas y el peso de la cultura fiscal. En conjunto, ambos factores crearon incentivos que 
Hernández Breña, W. Gerencialismo en un ambiente de regulaciones débiles y cultura fuerte: El caso de las Fiscalías Corporativas en Perú. Derecho y Ciencias Sociales. Octubre 2017. № 17. (Estudios actuales sobre la justicia penal) Pgs145-163 ISNN 1852-2971. Instituto de Cultura Jurídica y Maestría en Sociología Jurídica. FCJ y S. UNLP

espontáneamente crearon estilos de gestión no planificados guiados por los fiscales coordinadores.

De un lado, toda reglamentación tiene una intención directa: delimitar lo regulado a un determinado campo de acción y, al hacerlo, excluir aquello que no se debe o puede regular. Pero cuando la reglamentación es general,se corre el riesgo que los actores la interpreten o que busquen cubrir su vacíos en forma espontánea. Este fue el caso de las FC Corporativas. Su Reglamento priorizó una visión administrativa para la función del fiscal coordinador y dio menos peso a la coordinación de posiciones jurídicas.

Del otro lado, la cultura fiscal jugó un rol central en la creación de determinados incentivos y la alteración de prácticas cotidianas en la gestión que realizan los fiscales, lo cual insidiosamente desvalorizó lo corporativo. Sostenemos que son tres los rasgos de la cultura fiscal necesarios para entender tales incentivos y la alteración de las prácticas de gestión:la exacerbación del principio de autonomía y cómo esta introduce limitaciones a los posibles cambios de gestión; la jerarquía en el Ministerio Público como un factor de estructura y socializador que impulsa la sujeción y menos la coordinación; y la visión de política pública individualizada (y no transversal) que existe en los fiscales y que se da como consecuencia de la lógica de trabajar "caso por caso".

Ambos factores, reglas generales y cultura fiscal, dieron forma a los incentivos que produjeron los estilos espontáneos de gestión. Pero no se trata de incentivos economicistas en un sentido de costo y beneficio. Ningún fiscal rechazó la corporativización bajo el argumento que la lotización de actividades sea más eficiente. Por el contrario, son incentivos en reacción al grado de comunicación y proactividad de los fiscales coordinadores que, en el escenario de doble transición (corporativización de las fiscalías y la adopción del nuevo Código Procesal Penal), crearon prácticas nuevas que socavaron el principio anhelado de la eficiencia y que nunca pusieron por encima la calidad del trabajo fiscal.

Restringiéndonos a lo gerencial, una primera explicación al fracaso de la corporativización está en el abordaje incompleto de lo que Alfaro (2004) denomina los tres ejes básicos de todo proceso de mejora: organización, personas y tecnologías de la información y comunicación. Aun cuando las FC Corporativas parezcan haber operado bajo un escenario de gerencialismo, lo cierto es que el caso chileno con el que podemos comparar el caso peruano saca a relucir la limitada profundidad de las regulaciones operativas en nuestro país. 
Hernández Breña, W. Gerencialismo en un ambiente de regulaciones débiles y cultura fuerte: El caso de las Fiscalías Corporativas en Perú. Derecho y Ciencias Sociales. Octubre 2017. № 17. (Estudios actuales sobre la justicia penal) Pgs145-163 ISNN 1852-2971. Instituto de Cultura Jurídica y Maestría en Sociología Jurídica. FCJ y S. UNLP

Ahora limitando la respuesta a lo cultural, la creación de normas contrarias a la praxis cotidiana de fiscales y a su estructura como institución tiene escasa posibilidad de instaurar nuevos usos y quebrar prácticas de gestión enraizadas en una cultura en la que cada fiscal prefiere gestionar sus recursos en forma aislada bajo el interés de proteger una mal entendida autonomía.

Integrando ambas repuestas, podemos afirmar que la falta de regulaciones no lleva necesariamente a alcanzar la eficiencia. Toda regulación, débil o fuerte, genera incentivos nuevos que, en mayor o menor medida, pueden alejar la práctica de los objetivos planificados si no se toman en cuenta los factores culturales que propician la aparición de reglas informales. Igualmente, convertir a la cultura fiscal en una esencia inmutable crea barreas de cambio más fuertes que las que se derivan de la propia cultura fiscal.

El riesgo de tomar la eficiencia como eje único de la acción de los fiscales corre el riesgo de tropezarse con barreras mayores en la búsqueda de impacto sobre la corrupción, sobre todo si en el Perú solo un $4 \%$ de casos llega a juicio oral y recibe una sentencia que puede ser condenatoria o absolutoria. Aunque en un sistema distinto, Díez Ripollés (2004) critica al derecho penal español por orientarse a tutelar los presupuestos más esenciales para la convivencia, aun cuando solo con justicia no se logre lo que en forma conjunta debería alcanzarse alineando los objetivos del sistema penal con los de otros medios de control social (familia, escuela, vinculaciones comunitarias, medio laboral, relaciones sociales, opinión pública, etc.).

Sabiendo que no podemos poner en las FC Corporativas todo el peso de la lucha anticorrupción, tampoco podemos hacer de la eficiencia su objetivo mayor si es que no lo acompañamos de medidas que aseguren la calidad del trabajo fiscal y la adopción de nuevas prácticas que rompan con una cultura que en el mediano plazo puede socavar todos los beneficios de la celeridad u oralidad en un proceso penal.

\section{Referencias}

Alfaro, R. (2004). Visión integrada de la reforma procesal penal en Chile. IX Congreso Internacional del CLAD sobre la Reforma del Estado y de la Administración Pública, (págs. 1-8). Madrid. 
Hernández Breña, W. Gerencialismo en un ambiente de regulaciones débiles y cultura fuerte: El caso de las Fiscalías Corporativas en Perú. Derecho y Ciencias Sociales. Octubre 2017. No 17. (Estudios actuales sobre la justicia penal) Pgs145-163 ISNN 1852-2971. Instituto de Cultura Jurídica y Maestría en Sociología Jurídica. FCJ y S. UNLP

Binder, A. (s/f). La reforma de la justicia penal en América Latina como una política de largo plazo. En C. Villadiego (Ed.), La reforma a la justicia en América Latina: Las lecciones aprendidas (págs. 54-102). Friedrich Ebert Stiftung.

Centro de Estudios de la Justicia de las Américas. (2014). Evaluación de la implementación del Sistema Penal Acusatorio.

Ciocchini, P. (2013). Moldeando el problema y sus soluciones: los discursos técnicos sobre la demora en la administración de justicia penal. Crítica Jurídica, 36, 95-123.

Dasgupta, P., Hammond, P., \& Maskin, E. (1979). The implementation of social choice rules: Some general results on incentive compatibility. Review of Economics Studies, 46(2), 185216.

Díez Ripollés, J. L. (2004). El nuevo modelo penal de la seguridad ciudadana. Revista Electrónica de Ciencia Penal y Criminología, 06-03, 3-34.

Duce, M. (2008). El ministerio público en la reforma procesal penal en Améri-. Sistemas Judiciales, Revista 8: Posibilidades y límites de la comparación en los sistemas judiciales, 7, 65-82.

Garavano, G. (2004). La cultura y el sistema jurídico como condicionante de la Reforma Judicial. Ponencia en Congreso sobre Sistemas Judiciales Comparados.

González, C. (2016). Hacia un modelo organizacional del sistema de justicia penal en Latinoamérica. La influencia del management y del gerencialismo en esta reconfiguración. Nova Criminis, 7(11), 135-170.

Grzymala-Busse, A. (2010). The Best Laid Plans: The Impact of Informal Rules on Formal Institutions in Transitional Regimes. Studies in Comparative International Development, 45, 311-333.

Hammergren, L. (2003). Uses of empirical research in refocusing judicial reforms: Lessons from five countries. Washington: Banco Mundial.

León, R. (1996). Diagnóstico de la cultura judicial peruana. Lima: Academia de la Magistratura. 